\title{
STEADY CURRENTS INDUCED BY SEA WAVES PROPAGATING OVER A SLOPING BOTTOM
}

\author{
Erminia Capodicasa ${ }^{1}$ Pietro Scandura ${ }^{1}$ and Enrico Foti ${ }^{1}$
}

\begin{abstract}
A numerical model aimed at computing the mean velocity generated by a sea wave propagating over a sloping bottom, offshore the breaker line, is presented. The model is based on the assumption that the fluid domain can be partitioned into two boundary layers and a core region where at a first order of approximation the flow can be regarded as irrotational. The irrotational flow is computed by using a theory based on the assumption of small amplitude waves which allows both fully absorbed waves and partially reflected waves at the coastline to be considered. The distribution of the mean velocity is controlled by the ratio between the thickness of the boundary layer and the wave amplitude. When this ratio is small, the mean velocities are rather constant along the depth and a second boundary layer develops close to the bottom. In the case of fully reflected waves such boundary layer separates and the mean vorticity can be convected far from the bottom.
\end{abstract}

Keywords: sea waves; boundary layers; steady currents

\section{Introduction}

It is well known that the motion of the fluid particles under sea waves, apart a predominant oscillating component also presents a mean Lagrangian velocity known as mass transport. Under the assumption of irrotational flow, Stokes (1847) showed that such a mean velocity causes the migration of the fluid particles in the direction of wave propagation. This result is valid for waves propagating in an unbounded domain and in this particular case the mean Lagrangian velocity is also denoted as Stokes drift. For waves propagating in a bounded domain, as waves propagating towards the coast or waves in a laboratory wave flume, the mass conservation requires that a backward flow must be generated which balance the integral of the Stokes drift along the depth.

Although this steady flow is weak compared to the oscillating component, it plays an important role in the transport of sediment and pollutants in the sea environment; for such a reason in the past it has been the object of several studies. The experimental results of Bagnold (1947) showed important differences with the the irrotational wave theory as concerns the profiles of the steady current. Such discrepancies were explained by Longuet-Higgins (1953) for the case of small amplitude waves by introducing the effect of the viscosity.

The main effect of the viscosity is to induce a mean Reynolds stress in the boundary layers which generates a mean Eulerian velocity. Close to the bottom the mean Eulerian velocity is of second order in the wave slope and it persists outside the boundary layer. At the free surface it is the mean vorticity that is of the second order and it persists at the outer edge of the boundary layer.

The residual hydrodynamic quantities at the outer edge of the boundary layers affect the mean velocity in the core region by the mechanisms of diffusion and convection. Indeed, the distribution of the mean vorticity in the core region is described by a diffusion-convection equation in which the importance of the diffusive effects with respect to the convective ones is given by the ratio between the thickness of the boundary layer and the wave amplitude.

When a sea wave propagates over a constant depth and the wave attenuation because of energy dissipation can be neglected, the convective terms vanish and the mean velocity profiles can be computed according to the diffusion solution of Longuet-Higgins (1953). When the wave attenuation cannot be neglected and/or the water depth is not constant, the convective terms cannot be neglected unless the thickness of the boundary layer is larger or of the same order of magnitude of the wave amplitude. Usually, both in the field and in laboratory experiments the convective effects prevails over the viscous ones, apart close to the bottom where a second boundary layer develops in which the two effects have the same importance. The existence of a second boundary layer in an oscillating fluid was highlighted for the first time by Stuart (1966) during his study on the mean motion around a cylinder.

After the work of Longuet-Higgins (1953) many others have been carried out in order to analyze different aspects of the problem, as for example Dore (1977), Liu (1977) and Iskandarani and Liu (1991). Most of the previous works were aimed at studying the mass transport due to waves propagating over an horizontal bottom.

\footnotetext{
${ }^{1}$ Department of Civil and Environmental Engineering, University of Catania, Viale A. Doria 6, Catania, 95018, Italy
} 
The case of waves propagating over sloping bottom has important features that is interesting to analyze. When a sea wave propagates in water of decreasing depth it steepens until breaks. After breaking the mechanism which produces the steady flow is more complicated as the lost of regularity of the free surface causes the production of vorticity which is difficult to evaluate. However, thanks to studies such as Svendsen (1984), Stive and Wind (1986), much information are known as regards the mean current in the breaking region.

On the other hand few studies have been carried out in order to analyze the steady current outside the breaking region. Among these we mention the work of Hunt and Jones (1963) who developed formulas to compute the mean flow in the bottom boundary layer for the case of three-dimensional waves, without considering the flow far from the bottom. Recently, Blondeaux et al. (2002) developed a mathematical model to compute the mean flow induced by a sea wave propagating over a sloping bottom. The authors simplified the governing equations by introducing the shallow water approximations and therefore limitations on the ratio between water depth and wavelength.

The aim of the present work is to develop a mathematical model to describe the mean velocity in the region offshore from the breaker line without any limitation about the ratio between the water depth and the wavelength. The flow is assumed laminar but the Reynolds number is high enough such that the thickness of the boundary layers is small and therefore it is appropriate to consider the presence of a core region where at a first approximation the flow can be considered as irrotational.

\section{Formulation of the problem and numerical approach}

We consider a plane sloping beach and a sinusoidal wave propagating along the direction normal to the coastline as shown in Fig. 1. The wave has an angular frequency $\sigma^{*}$ and in deep water is characterized by an amplitude $a_{0}^{*}$ and a wavenumber $k_{0}^{*}$. Hereinafter a star is used to denote dimensional quantities.

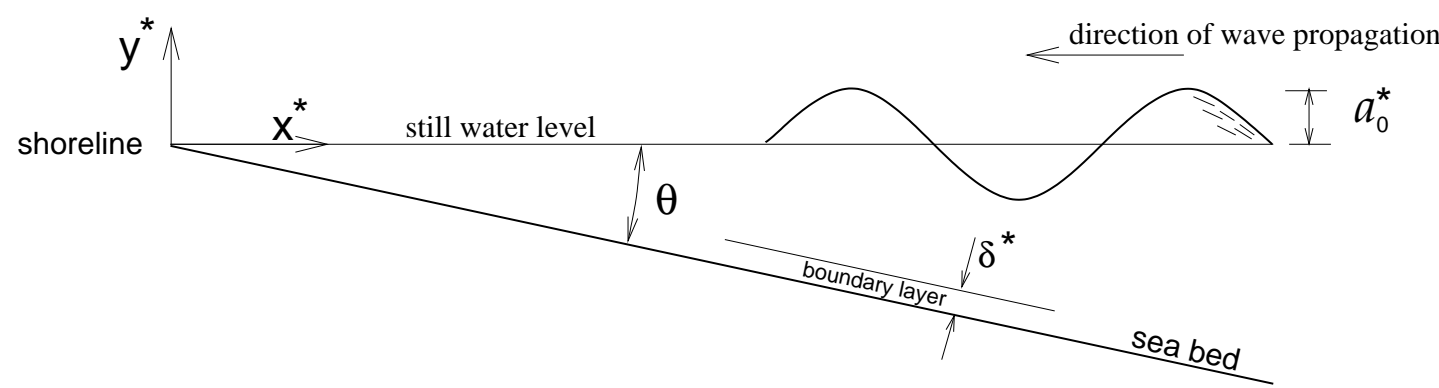

Figure 1. Sketch of the problem that shows a sea wave propagating towards the coast.

Since we consider waves of constant characteristics along the coast, the mean flow can be considered two-dimensional, therefore it is sufficient to introduce a reference system with the $x^{*}$ axis lying on the still water level, orthogonal to the coastline and directed offshore and with the $y^{*}$ axis vertical and directed upwards. The origin of the reference system is fixed at the intersection between the still water level and the sloping bottom which is described by the equation $y^{*}=-\beta x^{*}$, where $\beta$ is the slope of the beach. The problem is formulated in dimensionless form by introducing the following dimensionless variables:

$$
(x, y)=\left(x^{*}, y^{*}\right) k_{0}^{*}, t=t^{*} \sigma^{*},(u, v)=\frac{\left(u^{*}, v^{*}\right)}{\sigma^{*} a_{0}^{*}}, P=\frac{P^{*}}{\rho^{*} a_{0}^{*} \sigma^{* 2}} k_{0}^{*},
$$

where $t^{*}$ is the time, $\left(u^{*}, v^{*}\right)$ are the velocity components along the $x^{*}$ and $y^{*}$ axis respectively, $\rho^{*}$ is the density, $P^{*}$ is the dynamical pressure which is linked to the total pressure $p^{*}$ by the relation $p^{*}=P^{*}-\rho^{*} g^{*} y^{*}, g^{*}$ being the acceleration of gravity. The flow is described by the continuity and the momentum equations along with the boundary conditions. The boundary conditions are defined as follows: (1) the free surface is a material surface; (2) the fluid velocity vanishes at the bottom; (3) the stress vanishes at the free surface. After writing the governing equations and the boundary conditions in dimensionless form, we observe that the flow depends on the following three dimensionless parameters: 


$$
a_{0}=k_{0}^{*} a_{0}^{*}, \quad \delta=k_{0}^{*} \delta^{*}=k_{0}^{*} \sqrt{\frac{2 v^{*}}{\sigma^{*}}}, \quad \beta,
$$

where $v^{*}$ is the kinematic viscosity of the fluid. The parameter $a_{0}$ provides a measure of the importance of the nonlinearities. In the following we assume that this parameter is much smaller than one as it seems to be realistic for sea waves offshore the breaker line. The parameter $\delta$ is the dimensionless thickness of the boundary layer and it also provides a measure of the diffusive effects. This parameter takes values much smaller than one.

Since we have two parameters much smaller than one, all the variables can be expanded in a double power series as shown in the following for the velocity $u$ :

$$
u=u_{0}+a_{0} u_{1}+O\left(a_{0}^{2}\right) ; u_{0}=u_{00}+\delta u_{01}+O\left(\delta^{2}\right) ; u_{1}=u_{10}+\delta u_{11}+O\left(\delta^{2}\right) .
$$

We substitute (3) in the governing equations and in the boundary conditions and solve the problems at the different orders of approximation. At the first order of approximation the flow in the core region is irrotational, therefore all the variables depend on the dimensionless potential $\phi=\phi^{*} k_{0}^{*} /\left(\sigma^{*} a_{0}^{*}\right)$.

In this study the solution for the potential function has been computed according to Stoker (1947), who provided expressions for angles $\theta$ of the sloping bottom in the form $\theta=\pi /(2 n)$, where $n$ is an integer number. The expressions provide the potential of two standing waves which can be combined in order to obtain the potential of partially reflected waves at the coastline. In the following the irrotational velocity, given by the gradient of the potential function, will be written in the following form:

$$
(U, V)=(\hat{U}, \hat{V}) e^{i t},
$$

where $\mathrm{i}$ is the imaginary unit.

The irrotational flow does not satisfy the no slip condition at the bottom and the vanishing of the stress at the free surface. In order to satisfy such conditions, we introduce the boundary layers. At the bottom the boundary layer is better described in a reference system $\left(x^{\prime}, y^{\prime}\right)$ obtained by rotating the reference system $(x, y)$ in the clockwise direction until the $x$ axis coincides with the bottom.

At the first order of approximation we obtain that $P_{00}$ is constant in the direction orthogonal to the bottom and equal to that induced by the irrotational flow at the outer edge of the boundary layer. The velocity $u_{00}^{\prime}$ in the boundary layer is equal to that induced in a viscous fluid close to a flat plate by a sinusoidally time-oscillating pressure gradient (Stokes layer) and is given by

$$
u_{00}^{\prime}=\left(\hat{U}^{\prime}\right)_{y^{\prime}=0}\left(1-e^{-(1+i) \frac{y^{\prime}}{\delta}}\right) e^{i t} .
$$

The mean Eulerian velocity emerges by solving the problem at the order $a_{0}$. Here we report only the expression of the mean Eulerian velocity at the outer edge of the boundary layer (Mei et al. 2005),

$$
\bar{u}_{10}^{\prime}=-\frac{3}{4}\left(\hat{U}^{\prime} \frac{\partial \hat{U}^{\prime+}}{\partial x^{\prime}}\right)_{y^{\prime}=0}(1+i),
$$

where + denotes the conjugate of a complex number and the bar a time average.

The analysis of the free surface boundary layer is much more complicated than that of the bottom boundary layer as it requires the use of a curvilinear coordinate system which fits with the free surface (Longuet-Higgins 1953) or the use of a Lagrangian approach (Liu 1977) in order to be properly carried out. The vanishing of the stress at the free surface induces an oscillating velocity component of order $\delta$ which adds to the irrotational velocity and produces the following result:

$$
u_{00}=\left[(\hat{U})_{y=0}-\delta\left(\frac{\partial \hat{U}}{\partial y}\right)_{y=0}(1-i) e^{(1+i) y / \delta}\right] e^{i t} .
$$


At the second order of approximation a mean vorticity emerges which does not vanishes at the outer edge of the boundary layer where it assumes the following expression:

$$
\bar{\omega}_{10}=2 i\left(\frac{\partial \hat{U}^{+}}{\partial x} \frac{\partial \hat{V}}{\partial x}\right)_{y=0}
$$

Eqs. 6 and 8 provide two boundary conditions for the mean flow in the core region.

In the core region the second order mean vorticity $\bar{\omega}_{10}$ is described by a convection-diffusion equation (Longuet-Higgins 1953). Since the shape of the fluid domain is a circular sector, it is more appropriate to write this equation in a polar coordinate system,

$\left(u_{s r}+\frac{1}{r} \frac{\partial \bar{\psi}_{10}}{\partial \vartheta}\right) \frac{\partial \bar{\omega}_{10}}{\partial r}+\left(u_{s \vartheta}-\frac{\partial \bar{\psi}_{10}}{\partial r}\right) \frac{1}{r} \frac{\partial \bar{\omega}_{10}}{\partial \vartheta}=\varepsilon\left[\frac{1}{r} \frac{\partial}{\partial r}\left(r \frac{\partial \bar{\omega}_{10}}{\partial r}\right)+\frac{1}{r^{2}} \frac{\partial^{2} \bar{\omega}_{10}}{\partial \vartheta^{2}}\right],(9)$

where $u_{s r}$ and $u_{s \vartheta}$ are the velocity components of the Stokes drift along the $r$ and $\vartheta$ directions respectively; $\bar{\psi}_{10}$ is the stream function of the mean Eulerian velocity,

$$
\bar{u}_{10 r}=\frac{1}{r} \frac{\partial \bar{\psi}_{10}}{\partial \vartheta}, \bar{u}_{10 \vartheta}=-\frac{\partial \bar{\psi}_{10}}{\partial r},
$$

and $\varepsilon$ is a parameter which has the following expression $\varepsilon=\left(\delta / a_{0}\right)^{2} / 2$ and provides a measure of the importance of the viscous effects with respect to the convective ones.

The system of partial differential equations which describes the mean flow in the core region is completed by the equation that links the vorticity to the streamfuction,

$$
\bar{\omega}_{10}=-\left[\frac{1}{r} \frac{\partial}{\partial r}\left(r \frac{\partial \bar{\psi}_{10}}{\partial r}\right)+\frac{1}{r^{2}} \frac{\partial^{2} \bar{\psi}_{10}}{\partial \vartheta^{2}}\right]
$$

For field conditions and in many laboratory experiments, the parameter $\varepsilon$ in Eq. 9 is much smaller than one. Therefore the nonlinear terms are important and the solution must be determined by a numerical approach. In principle Eqs. 9, 10 and 11 should be solved in a domain which extends from a position $r=r_{1}$, offshore from the breaker line, up to $r=\infty$. However, since the solution must be computed by a numerical approach, the fluid domain must be truncated at a position $r=r_{2}>r_{1}$. Along the boundary of this domain appropriate boundary conditions must be introduced for the vorticity and the streamfunction. The bottom is a streamfunction, therefore here it is possible to fix arbitrarily $\bar{\psi}_{10}=0$. On the mean free surface the streamfunction can be obtained by imposing that the flux of the Eulerian velocity, computed by integrating the velocity from the bottom up to the free surface, must vanish. Following Blondeaux et al. (2002) we obtain

$$
\left(\bar{\psi}_{10}\right)_{y=0}=-\frac{i}{2}\left(\hat{U} \hat{V}^{+}\right)_{y=0} .
$$

As regards the vorticity, on the mean free surface we introduce the value determined at the outer edge of the free surface boundary layer given by Eq. 8. At the bottom, a predetermined value of the vorticity does not exists as the vorticity depends on the flow in the interior of the fluid domain. In order to determine a boundary condition for the vorticity at the bottom, as suggested by Roache (1972), we write Eq. 11 at $y=-\beta x$. Finally, it is also necessary to introduce a boundary condition along the side boundaries at $r=r_{1}$ and $r=r_{2}$. Unfortunately, there are not natural boundary conditions to be imposed in the previous locations as the computational domain has been truncated from the rest of the fluid region just along $r=r_{1}$ and $r=r_{2}$. However, the mathematics oblige us to select a boundary condition in order to close the problem. This is a common difficulty to many computational fluid dynamics problems when the boundaries are open and inflow and outflow along them may coexist. Although a general approach to determine an appropriate boundary condition does not exist, in these cases it is possible to state that the minimum requirement of such condition is that it must not influence the 
solution in particular far from the boundary (Sani and Gresho 1994). After considering different boundary conditions, along the side boundary we have introduced the following condition:

$$
\frac{\partial^{2} \bar{\psi}_{10}}{\partial r^{2}}=0 \text {. }
$$

As in Blondeaux et al. (2002) the appropriateness of this boundary condition has been checked for the case of standing waves by comparing the solution obtained by using Eq. 13 with that obtained by using the condition $\bar{\psi}_{10}=0$ along all the boundary after the two side boundaries were placed under a node or an antinode of the free surface.

The equations have been integrated numerically by a finite difference approach. The computational grid is uniform along the $r$ direction while along the $\vartheta$ direction it is stretched in order to cluster the grid points close to the bottom and close to the free surface where a large gradient exists.

The solution has been obtained by the method of the false transient, according to which a time derivative is added to the right hand side of Eq. 9 and the solution is advanced in time until a steady condition is attained. During the false transient the time advancement has been performed by a semiimplicit method. The convective terms and the derivatives with respect to $r$ of the viscous term have been evaluated explicitly while the derivatives with respect to $\vartheta$ of the viscous term have been evaluated implicitly.

\section{Discussion of the results}

The results in Fig. 2 show the trend of the mean velocity at the outer edge of the bottom boundary layer versus the distance $r$ from the shoreline, computed by Eq. 6, for different values of the reflection coefficient $K_{r}$. We observe that for a reflection coefficient equal to zero, which corresponds to waves fully absorbed at the coastline, the steady velocity is directed towards the coast and increases in this direction as the water depth decreases. For reflection coefficients larger than zero, the mean velocity oscillates between negative values for small reflection coefficients and between positive and negative values for large reflection coefficients.

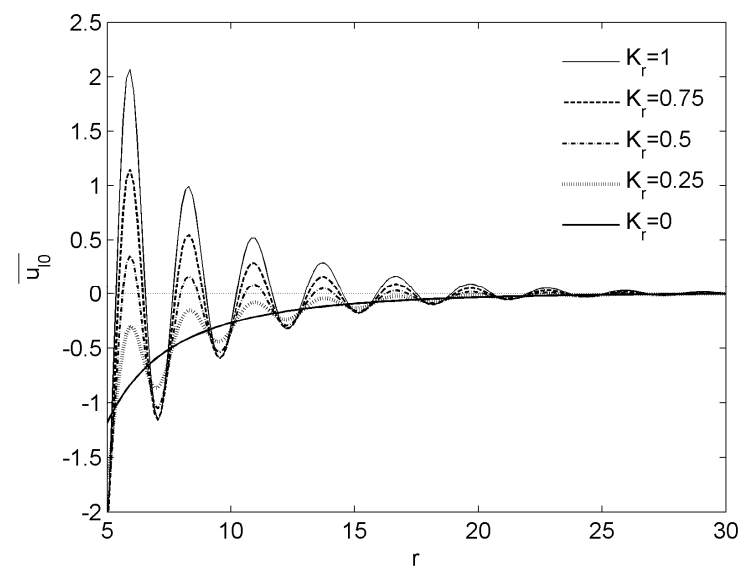

Figure 2. Trend of the mean velocity just outside the bottom boundary layer for different values of the reflection coefficient $\mathrm{K}_{\mathrm{r}}$. The angle $\theta$ of the beach is equal to $6^{\circ}$.

A first check on the numerical model has been performed by comparing the mean Lagrangian velocity with that computed by the diffusion solution of Longuet-Higgins (1953), which is valid for large values of the parameter $\varepsilon$. A very good agreement has been obtained between the two results as it can be observed in Fig. 3 for a slope of 1:30. The diffusion solution has been evaluated by using the local values of wavelength and wave-height estimated from the solution of the irrotational velocity field. The results also show that for a viscous-dominated flow the diffusion solution of Longuet-Higgins (1953) can be applied even on a bottom with a slope as small as 1:30. We did not investigate whether a good agreement can be achieved even in the case of a beach with a larger slope. Finally, in Fig. 3 it can be observed that the integral along the depth of the mean Lagrangian velocity vanishes as predicted by the theory. 


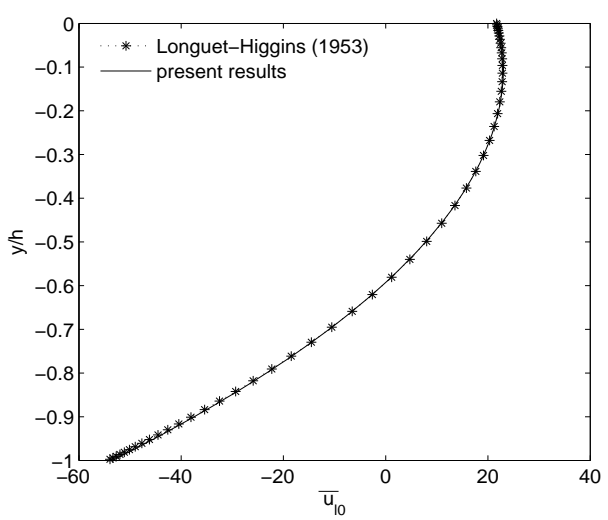

Figure 3. Mean Lagrangian velocity profile at $x=3.5$ along a beach with a slope of 1:30. The numerical results have been obtained for $\varepsilon>>1$.

A more significant validation of the numerical code has been carried out by comparing the numerical results with the experiments of Hwung and Lin (1990) who carried out measurements of the mean velocity at different stations along a bottom with a slope of 1:15. In Fig. 4 it can be observed a fairly good agreement between numerical results and experimental measurements. A discrepancy can be observed for the velocity profile closer to the shoreline, which is probably due to the turbulence induced by the wave breaking and advected offshore by the mass transport velocity.
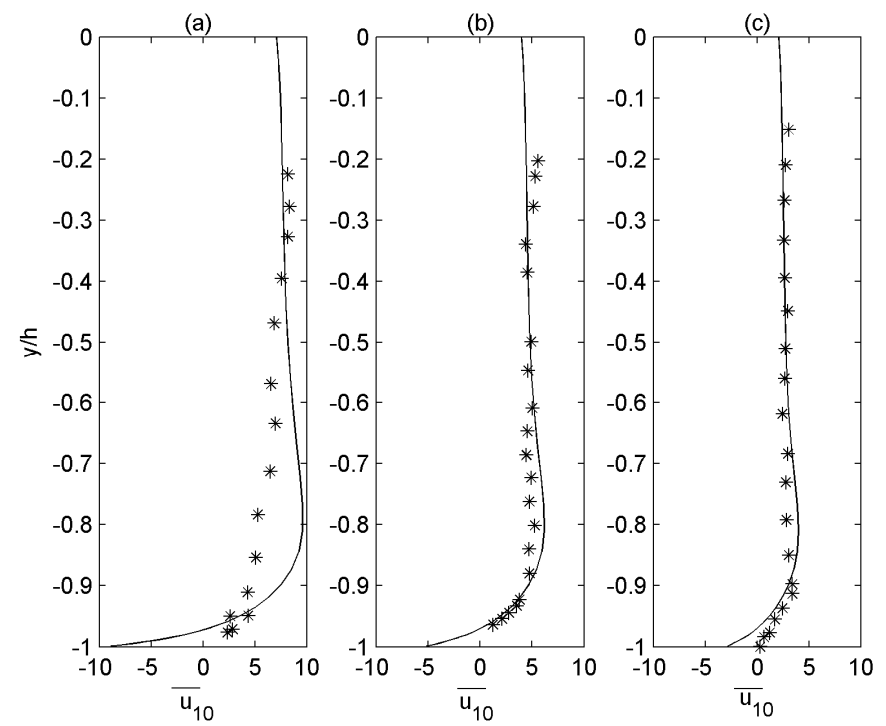

Figure 4. Comparison between the velocity profiles obtained by the present numerical model and those measured by Hwung and Lin (1990) for $T^{*}=1.41 \mathrm{~s}, a_{0}^{*}=5.3 \mathrm{~cm}, \varepsilon=2.7 \times 10^{-4}$, beach slope 1:15. (a) $h^{*}=9.8 \mathrm{~cm}$, (b) $h^{*}=13 \mathrm{~cm}$, (c) $h^{*}=17.4 \mathrm{~cm}$. Continuous line: numerical results; stars: experimental measurements.

Indeed, in the present model the flow is assumed laminar, therefore the effects of the turbulence cannot be reproduced. This explanation and the validity of the model are supported by the fairly good agreement that can be observed in Fig. 4 when positions not very close to the breaker line are considered.

We observe that far from the bottom the profiles are rather constant along the vertical, hence the inertial effects prevails over the viscous ones. Close to the bottom inertial and viscous effects are of the same order of magnitude, therefore a second boundary layer appears which is characterized by a thickness of the order of $\delta / a_{0}$.

In Fig. 5a the mean velocity profiles along the wave flume are reported for the same parameters of Hwung and Lin (1990) and in Fig. 5b for another case characterized by a larger value of the parameter $\varepsilon$. The viscous effects are larger in the right figure. The first three velocity profiles from the left are in the same locations as those shown in Fig. 4. For the smaller value of the parameter $\varepsilon$ (Fig. 5a), in shallow waters and close to the free surface the mean velocity is directed offshore but in deep water it becomes directed onshore. The tendency of the mean velocity to change sign decreases when the 
parameter $\varepsilon$ increases as shown in Fig. $5 b$. This result is due to the disappearance of the second boundary layer at the bottom for large viscous effects.
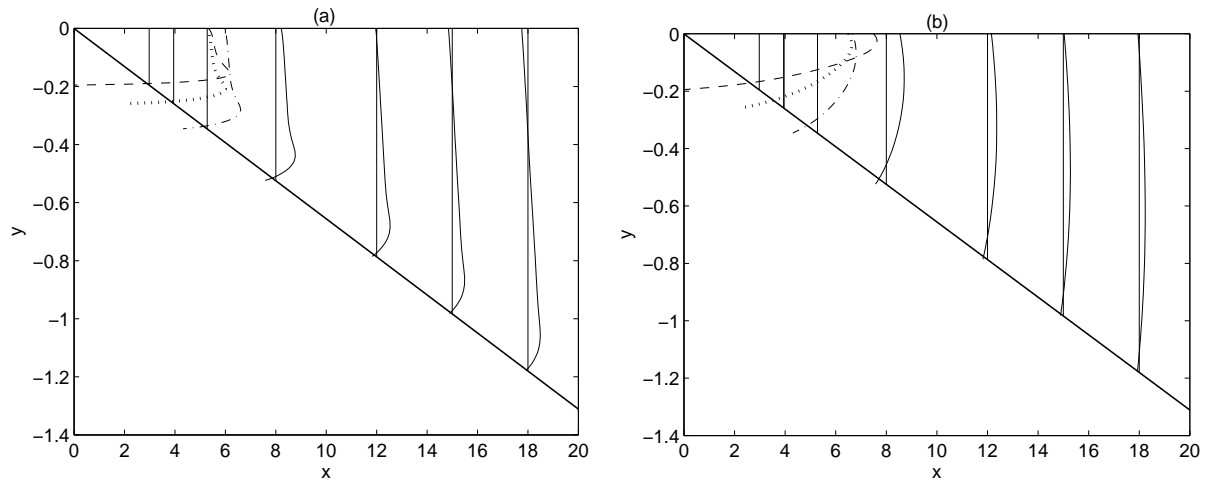

Figure 5. Mean velocity profiles along the channel for a beach with a slope of $1: 15$ (a); $\varepsilon=2.7 \times 10^{-4}$, (b) $\varepsilon=10^{-2}$.

The representation of the velocity field by streamlines for the case of Hwung and Lin (1990) is shown in Fig. 6a. We observe the presence of a saddle point at $(x, y)=(14,-0.14)$ which is due to the inversion of the velocity as previously explained. It can also be observed that the mean free surface is not a streamlines of the mean flow. On the other hand the mean free surface would be a streamline if the streamfunction of the mean Lagrangian velocity were considered.

In Fig. 6b, where the isolines of the vorticity are shown, we observe that large values of negative vorticity is present near the bottom, which witnesses the presence of a second boundary layer. Outside this layer the vorticity is rather low, only near the shoreline we observe a layer with moderate values of positive vorticity. This positive vorticity seems to be due to the interaction between the negative vorticity close to the bottom and the positive vorticity generated in the free surface boundary layer.
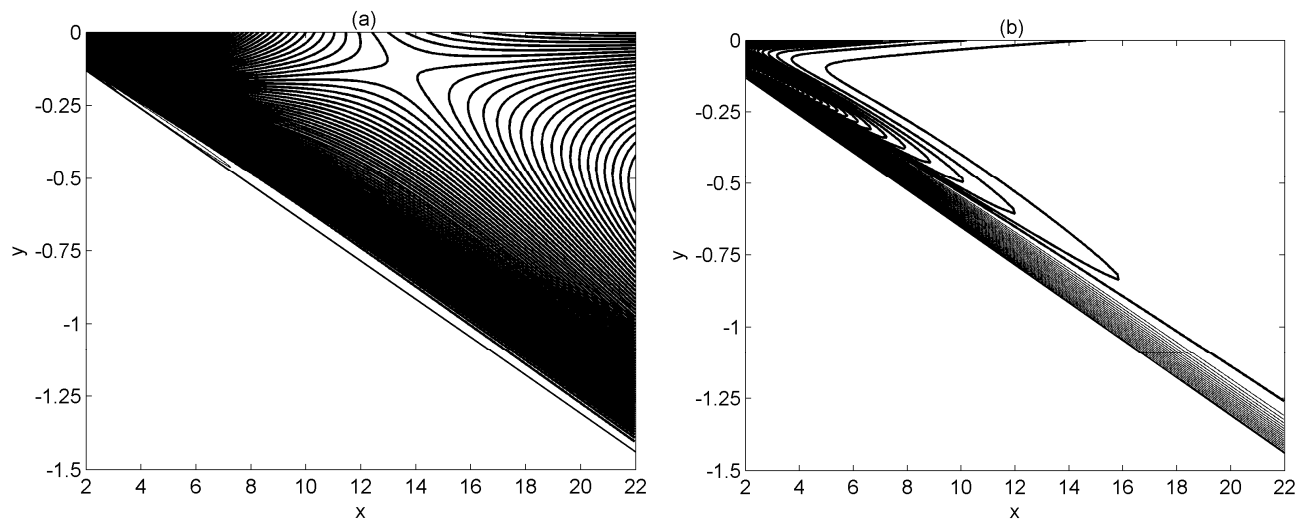

Figure 6. Streamlines (a) and vorticity (b) of the mean velocity for a slope of $1: 15$ and $\varepsilon=2.7 \times 10^{-4}$. Increment between two adjacent contour lines: (a) 0.01 (b) 1.5. Thick line: positive values, thin line: negative values.

When the value of the parameter $\varepsilon$ is increased, the vorticity appears to be distributed more uniformly in the fluid domain.

Up to now we have considered progressive waves completely absorbed at the coastline. In the opposite case of a progressive wave fully reflected at the coastline, a standing wave is generated which gives rise to a mean flow characterized by recirculating cells which depends on the steady streaming generated at the outer edge of the bottom boundary layer. We highlight that for standing waves both the streamfunction and the vorticity on the mean free surface are equal to zero. In this case if we choose the position of the side boundaries such that they coincide with the edges of the cells, the boundary condition for the streamfunction arises naturally and it can be written as $\bar{\psi}_{10}=0$. 

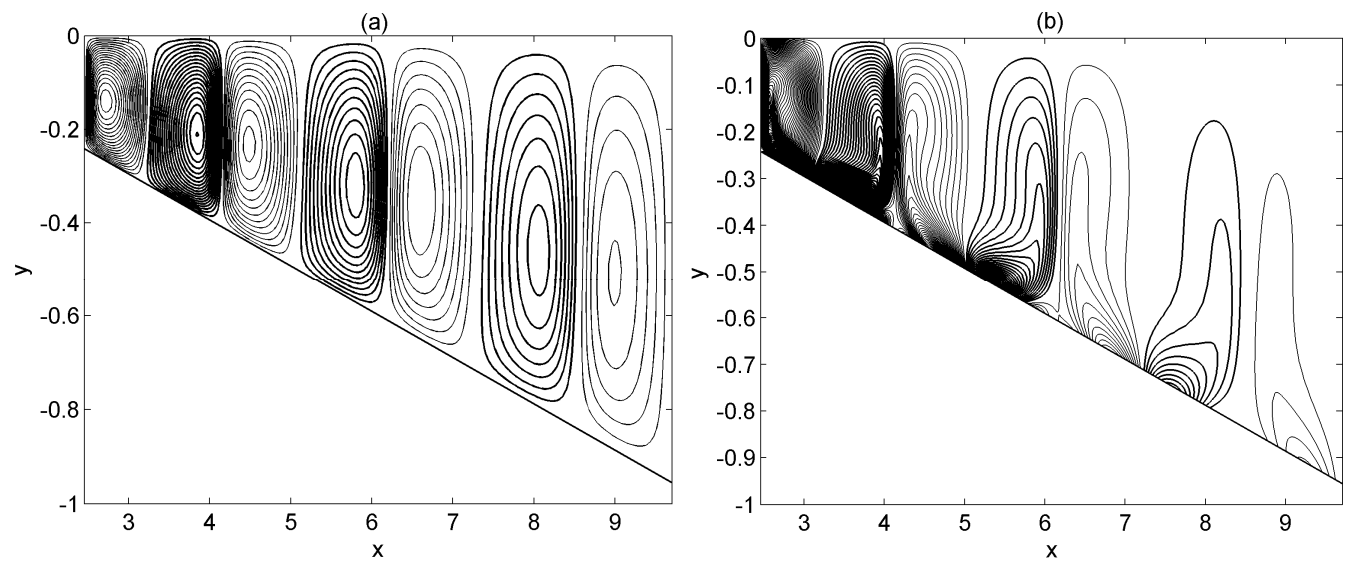

Figure 7. Streamlines (a) and vorticity (b) of the mean velocity for a fully reflected wave at the coastline propagating over a beach with a slope of $1: 10$, for $\varepsilon=0.01$. Increments between two adjacent contour lines: (a) 0.02 (b) 1.5. Thick line: positive values, thin line: negative values.

In Fig. 7 the streamlines and the vorticity contour plots for a fully reflected wave propagating on a bottom with a slope of $1: 10$ and for $\varepsilon$ equal to $10^{-2}$ are shown. We observe that positive and negative cells are present. The mean velocity is directed upwards under the antinodes of the free surface and downwards under the nodes. The vorticity generated on the bottom is convected upwards mainly under the antinodes, that is along the line of separation between a positive cell and the negative cell placed just offshore, as it clearly appears in Fig. 7b. This causes the centers of the previous two cells, in Fig. $7 \mathrm{a}$, moving the one towards the other as an increase of the vorticity causes a clustering of the streamlines.

In order to analyze the influence of an increase of the convective effects with respect to the viscous ones, we have repeated the previous simulation by using a value of $\varepsilon$ an order of magnitude smaller than the previous one. In such a case we observed that the negative and the positive cells interact causing the confinement of the positive cells close to the bottom and the merging of all the negative cells as it can be observed in Fig. 8a, where the results of a simulation carried out by introducing the condition $\bar{\psi}_{10}=0$ along the boundary is shown. Analogous results have been observed by using the boundary condition $\partial^{2} \bar{\psi}_{10} / \partial r^{2}=0$ at the side boundaries. We have carried out different simulations by changing the values of the parameters but in all the cases the negative cells were those that merged together. In order to gain insights about the mechanism that gives rise to the merging of the cells, we analyzed the flow field at an intermediate time during the false transient, when a steady condition has not attained yet.
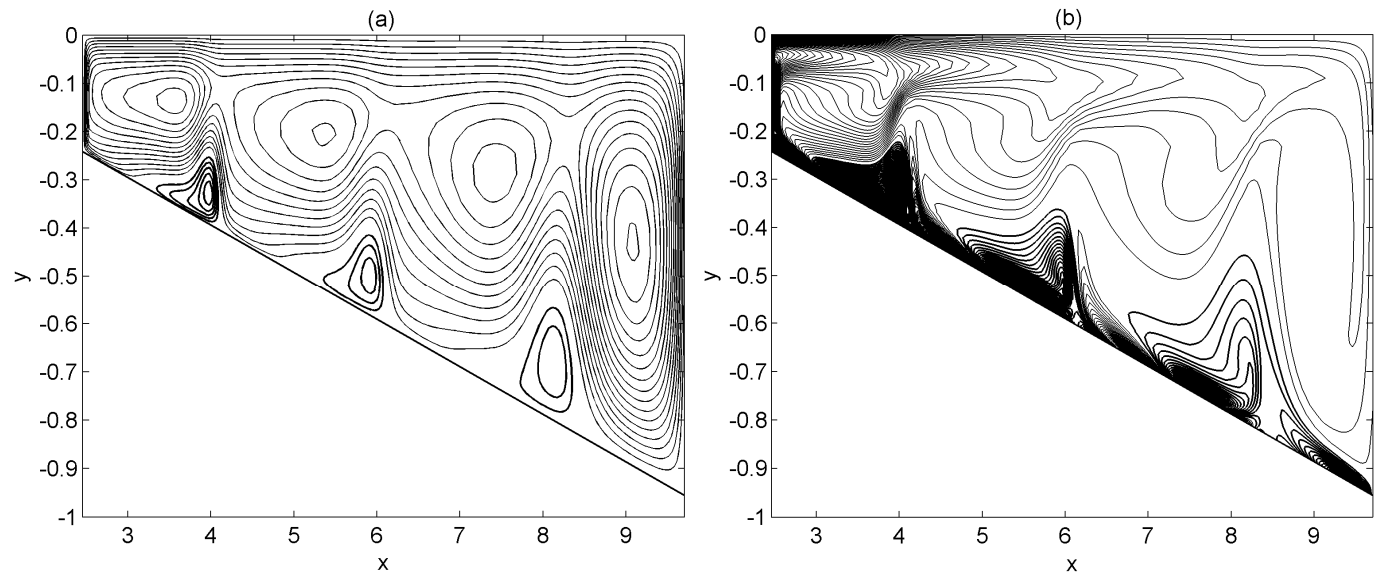

Figure 8. Streamlines (a) and vorticity (b) of the mean velocity for a fully reflected wave at the coastline propagating over a beach with a slope of $1: 10$, for $\varepsilon=0.001$. Increments between two adjacent contour lines: (a) 0.02 (b) 1.5. Thick line: positive values, thin line: negative values.

By carefully analyzing the vorticity field in Fig. 9, it appears that along the separation line between a negative cell and the positive cell placed just on the right, the vorticity of the negative cell is stronger 
than the vorticity of the positive cell, because the latter is placed in deeper water where the vorticity generated on the bottom is weaker.

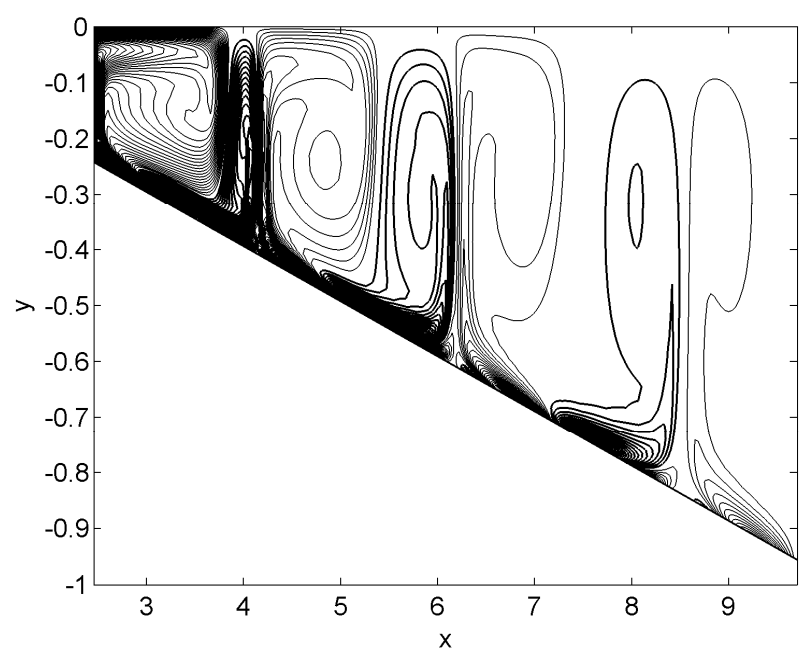

Figure 9. Vorticity for a fully reflected wave at the coastline on a beach with a slope of 1:10 and for $\varepsilon=0.001$. Increments between two adjacent contour lines: 1.5. Thick line: positive values, thin line: negative values. The results shown in this figure are pertinent to a time during the false transient when a steady condition has not attained yet.

The stronger negative vorticity induces a downward motion of the fluid in the positive cell which is incorporated by the negative cell. When the positive cell becomes small enough, the negative cell merges with the negative cell placed immediately offshore and starts to feed it with its vorticity. The process than propagates offshore until all the negative cells do not merge together.

\section{Conclusions}

In this paper the results of a model developed in order to compute the steady flow induced by a sea wave propagating over a sloping bottom, outside the surf zone, have been presented. The performance of the model has been checked by comparing the numerical results both with those of the theory and with those of experimental measurements.

In the case of a sea wave fully absorbed at the coastline, the mean velocity close to the bottom is directed onshore because of the steady streaming generated in the bottom boundary layer. Close to the free surface, in shallow waters the velocities are directed offshore, while in deep waters they are directed onshore. The inversion of the velocity produces a saddle point in the representation of the velocity field by streamlines.

For small values of the ratio between the thickness of the boundary layer and the wave amplitude, far from the bottom the velocity varies rather slowly, while large variations can be observed close to the bottom where a second boundary layer is generated which is adjacent to the Stokes layer.

In the case of fully reflected waves at the coastline, positive and negative recirculating cells are generated which produce upward ejections of vorticity that can reach the free surface. We have observed that when the convective effects are large, the positive and the negative cells interact such that the negative cells incorporate the fluid of the positive cells. The steady regime is characterized by positive cells confined close to the bottom and negative cells merged into one large cell.

\section{ACKNOWLEDGMENTS}

This paper has been partially funded by the HYDRALAB III in the framework of the Joint Research Activity SANDS (contract no. 022441(RII3)) and by the PRIN 2008 project 'Operative instruments for the estimate of coastal vulnerability in the presence of sandy beaches and also in the presence of coastal structures'.

\section{REFERENCES}

Bagnold, R.A. 1947. Sand movements by waves: some small scale experiments with sand of very low density, J. Inst. Civil Engrs, 27, 447-469.

Blondeaux, P., Brocchini, M. and G. Vittori. 2002. Sea waves and mass transport on a sloping beach, Proc. R. Soc. London A, 458, 2053-2082. 
Dore, B.D. 1977. On mass transport velocity due to progressive waves, Q. J. Mech. Appl. Math. 30(2), 157-173.

Hunt, J.N., and B. Johns. 1963. Currents induced by tides and gravity waves, Tellus, 15, 343-351.

Hwung, J.N., and C. Lin. 1990. The mass transport of waves propagating over a sloping bottom, Proc. $22^{\text {nd }}$ Int. Conf. Coastal Engineering, ASCE, pp. 544-556, Reston.

Iskandarani, M., and P.L.F. Liu. 1991. Mass transport in three dimensional water waves, J. Fluid Mech., 231, 417-437.

Liu, P.L.F. 1977. Mass transport in the free surface boundary layers, Coastal Engng, 1, 207-219.

Longuet-Higgins, M.S. 1953. Mass transport in water waves, Phil. Trans. R. Soc. Landon A, 245, 535581.

Mei, C.C., Stiassnie M., and D.K-P. Yue. 2005. Theory and applications of ocean surface waves. Part 2: Nonlinear Aspects, Advanced Series on Ocean Engineering 23. Singapore, World Scientific.

Roache, P.J. 1972. Computational fluid dynamics, Albuquerque, NM: Hermosa, 1972.

Sani, R.L., and P.M. Gresho. 1994. Résumé and remarks on the open boundary condition minisymposium, Int. J. Num. Meth. In Fluids, 18, 983-1008.

Stive, M.J.F., and H.G. Wind. 1986. Cross-shore mean flow in the surf zone, Coastal Engng, 10, 325340.

Stoker, J.J. 1947. Surface waves in water of variable depth, Quart. Appl. Math., 5, 1-54.

Stokes, G.G. 1847. On the theory of oscillatory waves, Trans. Camb. Phil. Soc., 8, 441-455.

Stuart, J.T. 1966. Double boundary layer in an oscillating viscous fluid, J. Fluid. Mech., 24, 673-687.

Svendsen, I.A. 1984. Mass flux and undertow in a surf zone, Coastal Engng., 8, 247-356 